\title{
Operations on Intuitionisitc Fuzzy Soft Sets Based on First Zadeh's Logical Operators
}

\author{
Nithya. A \\ M.Phil Scholar, KG College of Arts and Science, Coimbatore, Tamil Nadu, India
}

\begin{abstract}
In this paper, we have defined First Zadeh's intuitionistic fuzzy conjunction and intuitionistic fuzzy disjunction of two intuitionistic fuzzy soft sets. The author also defined some of their basic properties of intuitionistic fuzzy disjunction and conjunction with some examples.
\end{abstract}

KEY WORDS: Fuzzy soft sets, Intuitionistic fuzzy soft sets, first zadeh's intuitionistic fuzzy conjunction, first zadeh's intuitionistic disjunction

\section{INTRODUCTION}

The concept of the intuitionistic fuzzy was introduced in 1983 by Atanassov[1] as an extension of Zadeh's fuzzy set. All operations, defined over fuzzy sets were transformed for the case the intuitionistic fuzzy set case. This concept is capable of capturing the information that includes some degree of hesitation and applicable in various fields of research. For example, in decision making problems, particularly in the case of medial of medical diagnosis, sales analysis, new product marketing, financial services, etc. Atanassov et.al $[2,3]$ have widely applied theory of intuitionistic sets in logic programming, Szmidt and Kacprzyk [4] in group decision making, De et al [5] in medical diagnosis etc. therefore in various engineering application, intuitionistic fuzzy sets techniques have been more popular than fuzzy sets techniques in recent years. After defining a lot of operations over intuitionistic fuzzy sets during last ten years [6], in 2011, Atanassov [7,8] constructed two new operations based on the first Zadeh's intuitionistic fuzzy implication which are the first Zadeh's conjunction and disjunction, after that, in 2013, Atanassov [9] introduced the second type of Zadeh's conjunction and disjunction based on the Second Zadeh's intuitionistic fuzzy implication.
Another important concept that addresses uncertain information is the soft ser theory originated by Molodtsov [10]. This concept is free from the parameterization inadequacy syndrome of fuzzy set theory, rough set theory, probability theory. Molodtsov has successfully applied the soft set theory in many different fields such as smoothness of functions, game theory, operations research, Riemann integration, Perron integration and probability. In recent years, soft set theory has been received much attention since its appearance. There are many papers devoted to fuzzify the concept of soft set theory which leads tio a series of mathematical models such as fuzzy "soft sêt $[11,12,13,14,15]$, generalized fuizzy soft set $[16,17]$, possibility fuzzy soft set $[18]$ and so on. Thereafter, Maji and his coworker [19] introduced the notion of intuitionistic fuzzy soft set which is based on a combination of the intuitionistic fuzzy sets and soft set models and studied the properties of intuitionistic fuzzy soft ser. Later, a lot of extensions of intuitionistic fuzzy soft are appeared such as generalized fuzzy soft set [20], possibility intuitionsitc fuzzy soft set [21] etc.

In this paper, our aim is to extend the three new operations intrioduced by Atanassov to the case of intuitionistic fuzzy soft and study its properties. This paper is arranged in the following manner. In sections 2 , some definitions and notion about soft set, fuzzy soft set and intuitionistic fuzzy soft set and some properties of its. In section 3, the author discusses the two operations of intuitionistic fuzzy soft set such as First Zadeh's intuitionistc fuzzy conjunction and First Zadeh's intuitionistic fuzzy disjunction.

\section{Preliminaries}

In this section, some definitions and notions about soft sets and intuitionistic fuzzy soft set are given. 
Let $U$ be an initial universe, and $E$ be the set of all possible parameters under consideration with respect to $U$. The set of all subsets of $U$, i.e. the poer set of $U$ is denoted by $P(U)$ and the set of all intuitionistic fuzzy subsets of $U$ is denoted by IFU. Let $A$ be a subset of $E$.

\section{Definition 2.1}

A pair $(F, A)$ is called a soft set over $U$, where $F$ is a mapping given by $F: A \rightarrow P(U)$. In other words, a soft set over $U$ is a parameterized family of subsets of the universe $U$. For $e \in A, F(e)$ may be considered as the set of e-approxiamte elements of the soft set $(F, A)$.

\section{Definition 2.2}

Let $U$ be an initial universe set and $E$ be the set of parameters. Let $I F^{U}$ denote the collection of all intuitionistic fuzzy subsets of $U$. Let $A \subseteq E$ pair $(F, A)$ is called an intuitionistic fuzzy soft set over $U$ where $F$ is a mapping given by $F: A \rightarrow I F^{U}$.

\section{Definition 2.3}

Let $F: A \rightarrow I F^{U}$ then $F$ is a function defined as $F(\varepsilon)=\left\{x, \mu_{F(\varepsilon)}(x), v_{F(\varepsilon)}(x): x \in U\right\}$ where $\mu, v$ denote the degree of membership and degree of nonmembership respectively.

\section{Definition 2.4}

For two intuitionistic fuzzy soft sets $(F, A)$ and $(G, B)$ over a common universe $U$, we say that $(F, A)$ is an intuitionistic fuzzy soft subset of $(G, B)$ if

1. $A \subseteq B$ and

2. $F(\varepsilon) \subseteq G(\varepsilon)$ for all $\varepsilon \in A$. i.e $\mu_{F(\varepsilon)}(x) \leq \mu_{G(\varepsilon)}(x)$, $v_{F(\varepsilon)}(x) \leq v_{G(\varepsilon)}(x)$ for all $\varepsilon \in E$ and we write $(F, A) \subseteq(G, B)$.

In this case $(G, B)$ is said to be a soft super set of $(F, A)$.

\section{Definition 2.5}

Two soft sets $(F, A)$ and $(G, B)$ over a common universe $U$ are said to be soft equal if $(F, A)$ is a soft subset of $(G, B)$ is a soft subset of $(F, A)$.

\section{Definition 2.6}

Let $U$ be an initial universe, $E$ be the set of parameters, and $A \subseteq E$.

1. $(F, A)$ is called a relative null soft set ( with respect to the parameter set A), denoted by $\phi_{A}$, if $F(a)=\phi$ for all $a \in A$.

2. $(G, A)$ is called a relative null soft set ( with respect to the parameter set A), denoted by $U_{A}$, if $G(e)=U$ for all $e \in A$.

\section{Definition 2.7}

Let $(F, A)$ and $(G, B)$ be two intuitionistic fuzzy soft sets over the same universe $U$. Then the union of $(F, A)$ and $(G, B)$ is denoted by ' $(F, A) \cup(G, B)$ ' ' and is defined by $(F, A) \cup(G, B)=(H, C)$, where $C=A \cup B$ and the truth membership, falsity membership of $(H, C)$ are as follows:

$$
H(\varepsilon)=\left\{\begin{array}{cr}
\left\{\mu_{F(\varepsilon)}(x), v_{F(\varepsilon)}(x): x \in U\right\} & , \text { if } \varepsilon \in A-B \\
\left\{\mu_{G(\varepsilon)}(x), v_{G(\varepsilon)}(x): x \in U\right\} & , \text { if } \varepsilon \in B-A \\
\left\{\max \left(\mu_{F(\varepsilon)}(x), \mu_{G(\varepsilon)}(x)\right), \min \left(v_{F(\varepsilon)}(x), v_{G(\varepsilon)}(x)\right): x \in U\right\}, & \text { if } \varepsilon \in A \cap B
\end{array}\right.
$$

Where $\mu_{H(\varepsilon)}(x)=\max \left(\mu_{F(\varepsilon)}(x), \mu_{G(\varepsilon)}(x)\right)$ and $v_{H(\varepsilon)}(x)=\min \left(v_{F(\varepsilon)}(x), v_{G(\varepsilon)}(x)\right)$.

\section{Definition 2.8}

Let $(F, A)$ and $(G, B)$ be two intuitionistic fuzzy soft sets over the same universe $U$ such that $A \cap B \neq 0$. Then the intersection of $(F, A)$ and $(G, B)$ is denoted by ' $(F, A) \cap(G, B)$ ' and is defined by $(F, A) \cap(G, B)=(K, C)$, where $C=A \cap B$ and the truth membership, falsity membership of $(K, C)$ are related to those of $(F, A)$ and $(G, B)$ by: 
International Journal of Trend in Scientific Research and Development (IJTSRD) ISSN: 2456-6470

$$
K(\varepsilon)=\left\{\begin{array}{cr}
\left\{\mu_{F(\varepsilon)}(x), v_{F(\varepsilon)}(x): x \in U\right\} & , \text { if } \varepsilon \in A-B \\
\left\{\mu_{G(\varepsilon)}(x), v_{G(\varepsilon)}(x): x \in U\right\} & , \text { if } \varepsilon \in B-A \\
\left\{\min \left(\mu_{F(\varepsilon)}(x), \mu_{G(\varepsilon)}(x)\right), \max \left(v_{F(\varepsilon)}(x), v_{G(\varepsilon)}(x)\right): x \in U\right\}, & \text { if } \varepsilon \in A \cap B
\end{array}\right.
$$

Where $\mu_{K(\varepsilon)}(x)=\min \left(\mu_{F(\varepsilon)}(x), \mu_{G(\varepsilon)}(x)\right)$ and $v_{K(\varepsilon)}(x)=\max \left(v_{F(\varepsilon)}(x), v_{G(\varepsilon)}(x)\right)$.

\section{Definition 2.9}

Let $(F, A)$ and $(G, B)$ be two intuitionistic fuzzy soft sets $s$ over $(U, E)$. We define the First Zadeh's intuitionistic fuzzy soft set $\operatorname{implication}(F, A) \underset{z, 1}{\rightarrow}(G, B)$ is defined by $(F, A) \underset{Z, 1}{\longrightarrow}(G, B)=\left(\min \left\{v_{F(\varepsilon)}(x), \max \left(\mu_{F(\varepsilon)}(x), \mu_{G(\varepsilon)}(x)\right)\right\}, \max \left(\mu_{F(\varepsilon)}(x), v_{G(\varepsilon)}(x)\right)\right)$.

\section{Operations on intuitionistic Fuzzy Soft Sets Example 3.1.2}

Based on First Zadeh's Logical Operators Let $U=\{a, b, c\}$ and $E=\left\{e_{1}, e_{2}, e_{3}, e_{4}\right\}$,

3.1 First Zadeh's conjunction of intuitionistic $A=\left\{e_{1}, e_{2}, e_{4}\right\} \subseteq E, B=\left\{e_{1}, e_{2}, e_{3}\right\} \subseteq E$
Fuzzy Soft Sets

\section{Definition 3.1.1}

Let $(F, A)$ and $(G, B)$ be two intuitionistic fuzzy soft sets $s$ over $(U, E)$. We define the First Zadeh's intuitionistic fuzzy conjunction of $(F, A)$ and $(G, B)$ as the intuitionistic fuzzy soft set $(H, C) \operatorname{over}(U, E)$, written as $(F, A) \widetilde{\wedge}_{Z, 1}(G, B)=(H, C)$, re where $C=A \cap B \neq \phi$ and for all $\varepsilon \in C, x \in U$,

$$
\begin{aligned}
& A \cap B \neq \phi \text { and for all } \varepsilon \in C, x \in U, \\
& \mu_{H(\varepsilon)}(x)=\max \left(\mu_{F(\varepsilon)}(x), \mu_{G(\varepsilon)}(x)\right) \\
& v_{H(\varepsilon)}(x)=\min \left\{v_{F(\varepsilon)}(x), \max \left(\mu_{F(\varepsilon)}(x), v_{G(\varepsilon)}(x)\right)\right\} \text { elo } C=A \cap B \neq\left\{e_{1}, e_{2}\right\}
\end{aligned}
$$

$$
\begin{aligned}
& (F, A)=\left\{\begin{array}{l}
F\left(e_{1}\right)=\{(a, 0.2,0.4),(b, 0.5,0.3),(c, 0.6,0.1)\}, \\
F\left(e_{2}\right)=\{(a, 0.3,0.5),(b, 0.3,0.3),(c, 0.7,0.2)\}, \\
F\left(e_{4}\right)=\{(a, 0.4,0.5),(b, 0.1,0.8),(c, 0.4,0.6)\}
\end{array}\right\} \\
& (G, B)=\left\{\begin{array}{l}
G\left(e_{1}\right)=\{(a, 0.6,0.2),(b, 0.4,0.2),(c, 0.4,0.4)\}, \\
G\left(e_{2}\right)=\{(a, 0.2,0.5),(b, 0.1,0.2),(c, 0.2,0.6)\}, \\
G\left(e_{3}\right)=\{(a, 0.1,0.5),(b, 0.5,0.3),(c, 0.8,0.2)\}
\end{array}\right\}
\end{aligned}
$$

